\title{
BEAM DYNAMICS STUDIES FOR A CTF3 PROPOSED INJECTOR*
}

\author{
F.Zhou ${ }^{\dagger}$, D.Cline, UCLA, Los Angeles, CA 90095, USA
}

\begin{abstract}
A CTF3 drive beam injector for the nominal stage is proposed, which includes thermionic DC gun, three SubHarmonic Bunchers (SHBs), one prebuncher, one 6-cell travelling wave buncher and two accelerating structures. Its beam dynamics is simulated by PARMELA. It is shown that all important parameters, e.g. satellite charge, bunch length and normalized transverse emittance can be met with the design goals if about $400 \mathrm{~kW}$ RF power for 3 SHBs is available. In addition, the beam dynamics of the injector for the initial stage has been simulated.
\end{abstract}

\section{INTRODUCTION}

A new phase of the Compact Linear Collider (CLIC) Test Facility (CTF3) has been approved at CERN to demonstrate and test many critical components of the CLIC project [1]. The CTF3 drive beam linac will accelerate a 1.54 us long electron pulse of $3.5 \mathrm{~A}$ in a fully beam-loaded linac. Although the linac operates with a RF frequency of $3 \mathrm{GHz}$, the electrons are bunched with 1.5 $\mathrm{GHz}$ and the bunches are shifted from even to odd S-band buckets and vice-versa every $140 \mathrm{~ns}$. To provide this beam an injector is proposed consisting of a thermionic DC gun, three $1.5 \mathrm{GHz}$ subharmonic bunchers, one $3 \mathrm{GHz}$ prebuncher, one 6-cell travelling wave buncher and two travelling wave accelerating structures. Its beam dynamics has been extensively simulated with PARMELA. It is shown that all design requirements could be met if about $400 \mathrm{~kW}$ RF power for the subharmonic bunchers is available. The drive beam linac will be commissioned in two phases; the initial phase will operate without the subharmonic bunching. The injector configuration and beam dynamics for this phase are presented as well.

\section{PROPOSAL OF BUNCHING SYSTEM}

The bunching system consists of a $3 \mathrm{GHz}$ prebuncher and a travelling wave tapered phase velocity buncher accelerator. The one standing wave prebuncher needs about $30 \mathrm{kV}$ for optimum bunching. The distance between the prebuncher and the buncher is optimized to $8.5 \mathrm{~cm}$. For optimum bunching, many versions of the buncher configurations have been extensively investigated. With comparisons, a 6-cell travelling wave accelerator with tapered phase velocity is adopted. It is shown that the cells in the buncher need to start at a phase velocity of $0.7 \mathrm{c}_{\mathrm{o}}$ gradually increasing up to $0.81 \mathrm{c}_{\mathrm{o}}$. Further, the iris radius and accelerating gradients in the 6 cells are

\footnotetext{
*work was done at CERN
}

†zhouf@sun2.bnl.gov optimized. Table 1 gives the optimization results. It is shown that version 3 is the best. Its satellite charge ratio is smaller, $5.0 \%$, and not too sensitive to the accelerating gradient. In addition, simulations show that its transverse HOM mode frequency is well separated from the fundamental accelerating mode for this version [2].

Table 1: comparison of different buncher structures

\begin{tabular}{|l|c|c|}
\hline \multicolumn{1}{|c|}{$\begin{array}{l}\text { Iris radius } \text { (cm) } \\
\text { in } 6 \text { cells }\end{array}$} & $\begin{array}{l}\text { Satellite } \\
\text { (sate./main) }\end{array}$ & $\begin{array}{l}\text { RF power } \\
\text { for 3shbs }\end{array}$ \\
\hline $\begin{array}{l}1.70 / 1.52 / 1.52 / 1.52 / \\
1.52 / 1.52 / 1.52 /\end{array}$ & $6.0 \%$ & $320 \mathrm{~kW}$ \\
\hline $\begin{array}{l}2.30 / 2.33 / 2.36 / 2.40 / \\
2.43 / 2.46 / 2.50 /\end{array}$ & $6.5 \%$ & $400 \mathrm{~kW}$ \\
\hline $\begin{array}{l}1.7 / 2.12 / 2.16 / 2.20 / \\
2.24 / 2.28 / 1.7\end{array}$ & $5.0 \%$ & $400 \mathrm{~kW}$ \\
\hline
\end{tabular}

\section{BEAM DYNAMICS OF THE INJECTOR WITH NOMINAL STAGE}

Through many simulations and comparisons, one injector for CTF3 has been proposed. Its layout is schematically shown in Figure 1. It is composed of a 140 $\mathrm{keV}$ thermionic Gun, three $1.5 \mathrm{GHz}$ SHBs, one $3 \mathrm{GHz}$ prebuncher and a 6-cell travelling wave (TW) tapered phase velocity buncher as described in section 2 and two 32-cell TW accelerating structures. All components downstream of the gun are embedded in solenoid field.

\subsection{Longitudinal beam dynamics}

The simulations are started from the gun exit. There the kinetic energy is $140 \mathrm{keV}$ and the normalized emittance is assumed to be $5 \pi \cdot \mathrm{mm} \cdot \mathrm{mrad}$. A total of 6000 input particles with the range of $6 \mathrm{~S}$-band cycles are started for the simulations. Beam current at the gun exit is $5.7 \mathrm{~A}$ in order to guarantee the current of $3.5 \mathrm{~A}$ after the bunching.

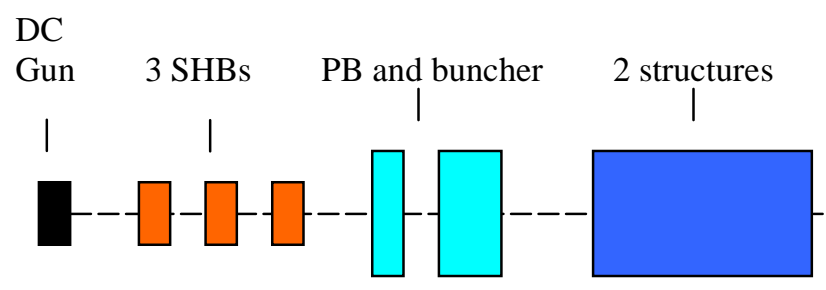

Figure 1: Schematic Layout of the injector

The main objective for the simulations is to make the satellite charge (in a $20^{\circ} \mathrm{S}$-band window) less than $5 \%$ 
of the main bunch (also in a $20^{\circ}$ window) in order to get maximum RF power production efficiency and minimum beam losses. The satellite signal is defined as $\delta=\frac{N_{1}}{N_{2}}$, where $\mathrm{N}_{1}$ is the particle number of the satellite within the range of a $20^{\circ}$ window, $N_{2}$ is the particle number of the main bunch within the range of a $20^{\circ}$ window. It is well known that the fewer particles in the satellite bucket, the higher RF power for 3 SHBs will be needed. The high RF power for $3 \mathrm{SHBs}$ implies high costs for the $1.5 \mathrm{GHz}$ RFsources. Thus, the needed RF power for SHBs should be as low as possible while the satellite charge is controlled below $5 \%$ of the main bunch. Another point is to make the bunch length as short as possible while the satellite charge is still controlled below 5\% of the main bunch $[3,4,5]$.

The beam dynamics with $400 \mathrm{~kW}$ RF power for $3 \mathrm{SHBs}$ are calculated by PARMELA. The phase space projections at the end of the injector are shown in Figures 2.

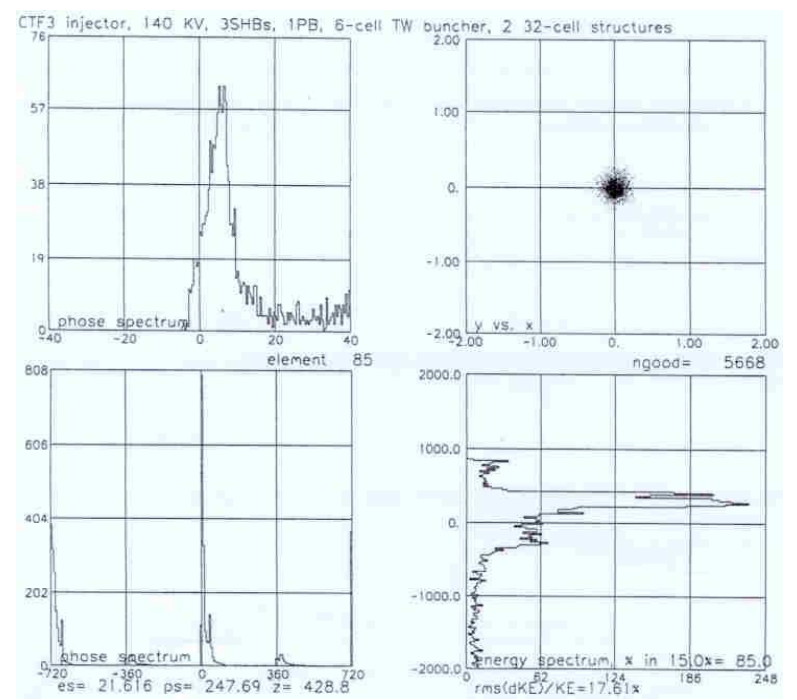

Figure 2: Beam spectrum with $400 \mathrm{~kW}$ for $3 \mathrm{SHBs}$

It is shown that its micro-bunch width at the end of the injector is near 10 ps (FWHM) and about $70 \%$ particles are captured in a $20^{\circ}$ window. A small fraction of particles are in the tail of the main bunch and outside of the $20^{\circ}$ window, and these particles will be cut off by scrapers in a dispersive section downstream of the injector. The charge in the satellite bucket is only about $5 \%$ of the main bunch.

\subsection{Transverse beam dynamics}

Two accelerating structures start at about $200 \mathrm{~cm}$ of longitudinal position along the beam line (calculating from the gun exit). Here, the emittance change at the end of injector is measured, as shown in Figure 3a, with different solenoid field in the two structures as shown in Figure $3 \mathrm{~b}$. The emittance may become lower if the tail in the bunch is cut off.
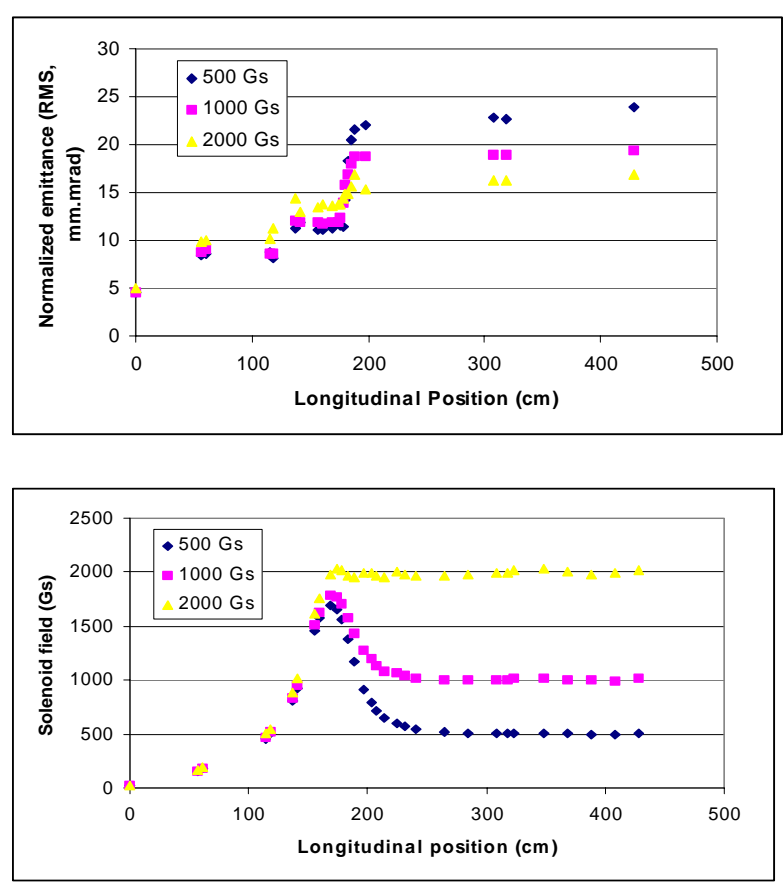

Figure 3: The emittance and solenoid field with longitudinal position

It is shown that the emittance difference with different solenoid field is not too large. However, beam envelopes with different solenoid field settings are different, as shown in Figure 4. It can be seen that the beam envelope with 500 Gauss is larger than that with 2000 Gauss by a factor of 2, however, the beam envelope with 1000 Gauss is close to the one with 2000 Gauss. The beam size at the injector with different solenoid field is shown in Figure 5. It is clear that the beam sizes with both 1000 Gauss and 2000 Gauss are smaller than the beam size with 500 Gauss field. Further comparing beam sizes of 1000 Gauss and 2000 Gauss, it is found that there are more halo particles with 2000 Gauss. In addition, the bunch length with 1000 Gauss is better than the one with 2000 Gauss. Considering these points, 1000 Gauss solenoid field is used for our final simulations.

\section{BEAM PARAMETERS FOR INITIAL STAGE}

CTF3 injector has three steps. The last two steps are initial and nominal stages. It is required to have same main elements for both stages. In the initial stage, SHBs are not necessary. The beam parameters are simulated when three SHBs are switched off in the layout of the injector with nominal stage. Only after optimizing the gradient of the buncher and the phases of both buncher and accelerating structures, the design goals of the CTF3 injector with initial stage can be met. Phase space projections at the injector output are shown in Figure 6. It is shown that all beam parameters can be met with the requirements. 

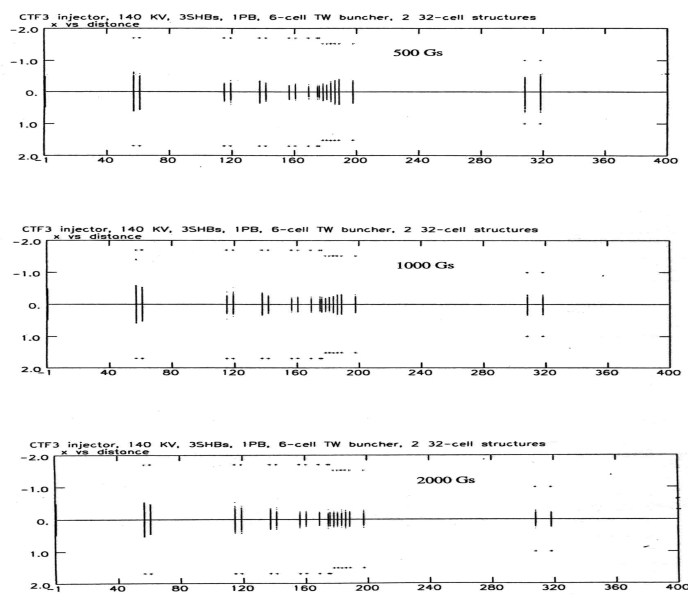

Figure 4: Beam envelope vs different solenoid field
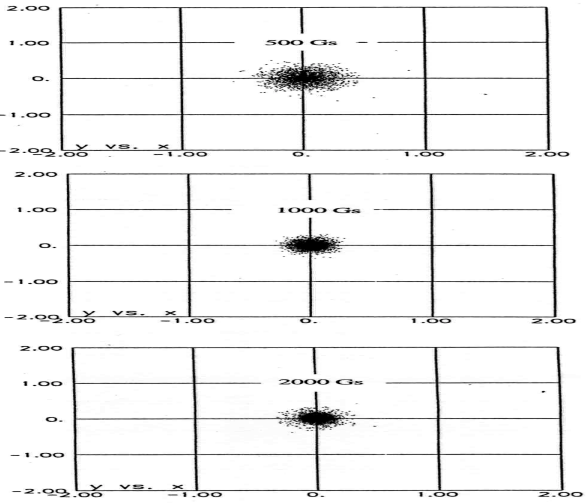

Figure 5: Beam sizes vs solenoid field

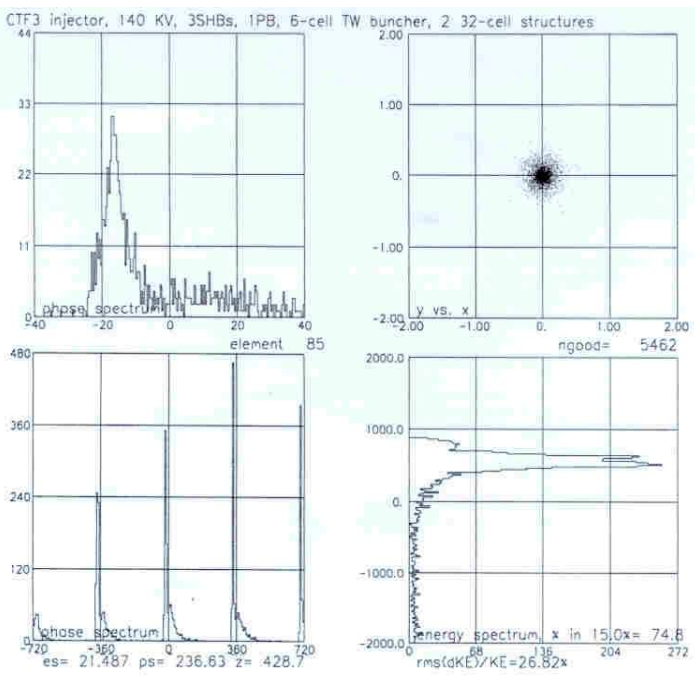

Figure 6: Spectrum with initial stage

\section{CONCLUSION}

Two schemes of CTF3 injectors been investigated at CERN. One is to use SHBs with same frequency. The other is to use SHBs with cluster frequencies [6]. The beam dynamics of the CTF3 injector with three SHBs using same frequency, one prebuncher and one TW buncher and two structures have been presented in this paper. Its injector with initial stage can be switched to the nominal stage when three SHBs are installed in between gun exit and prebuncher in the initial stage. Its main beam parameters for the initial and nominal stages are compared with the required performance, as summarized in Table 2. It is shown that all key parameters except of the final energy can be met when about $400 \mathrm{~kW}$ RF power for $3 \mathrm{SHBs}$ is available. The final energy can be increased by adding a third accelereating structure fed by the output coupler of the buncher. This is possible since the RFpower transmitted through the buncher will be barely attenuated.

Table 2: Comparison between the simulations and the requirements

\begin{tabular}{|l|c|c|c|}
\hline & $\begin{array}{l}\text { Initial } \\
\text { stage }\end{array}$ & $\begin{array}{l}\text { Nominal } \\
\text { stage }\end{array}$ & Required \\
\hline $\begin{array}{l}\text { RF power for 3 } \\
\text { SHBs (kW) }\end{array}$ & --- & $\mathbf{4 0 0}$ & $<\mathbf{5 0 0}$ \\
\hline Satellite & --- & $5.0 \%$ & $<5.0 \%$ \\
\hline $\begin{array}{l}\text { Bunch length } \\
\text { (FWHM, ps) }\end{array}$ & 10 & 10 & $<12$ \\
\hline $\begin{array}{l}\text { Bunch length } \\
\text { (FW, ps) }\end{array}$ & 20 & 20 & --- \\
\hline Energy (MeV) & 20 & 20 & 26 \\
\hline $\begin{array}{l}\text { Energy spread } \\
\text { (FWHM, MeV) }\end{array}$ & 0.25 & 0.25 & 0.5 \\
\hline $\begin{array}{l}\text { Charge/bunch } \\
\text { (nC) }\end{array}$ & 2.30 & 2.51 & 2.33 \\
\hline $\begin{array}{l}\text { Normalized } \\
\text { RMS emittance } \\
\text { (um.rad) }\end{array}$ & 20 & 20 & $<100$ \\
\hline
\end{tabular}

\section{ACKNOWLEDGEMENTS}

We would like to thank R.Miller and D.Yeremian for their helpful discussions and stimulating suggestions. Their experience was particular helpful for our simulations. Special thanks to R.Miller for providing the preliminary scheme with 2 SHBs. We further would like to thank Lars Thorndahl for his study of the buncher RF parameters. Thanks should also be given to Louis Rinolfi for his interest in these simulations.

\section{REFERENCES}

[1] CLIC study team, Proposals for future CLIC studies and a new CLIC Test Facility (CTF3), CLIC Note 402, 1999.

[2] L.Thorndahl, private communication, 2000.

[3]L.Rinolfi (ed.), Proceeding of CLIC collaboration meeting between CERN/SLAC, May 2000.

[4] F.Zhou, H.Braun, Optimization of the CTF3 Injector with 2 SHBs and 3 SHBs, CTF3 Tech Note 00-01, 2000.

[5] R.Corsini, CTF3 meeting minutes, May 2000.

[6]D.Yeremian, CERN/SLAC collaboration meeting, October 2000. 